28

IMPACT OF OPIATE ANALGESIA ON PLATELET REACTIVITY IN PATIENTS PRESENTING FOR PRIMARY PERCUTANEOUS CORONARY INTERVENTION

${ }^{1}$ Mohamed Farag*, 'Manivannan Srinivasan, ${ }^{2}$ Diana A Gorog. ${ }^{1}$ East and North Hertfordshire NHS Trust; ${ }^{2}$ Imperial College; *Presenting Author

\subsection{6/heartjnl-2016-309890.28}

Background ST-elevation myocardial infarction (STEMI) is caused predominantly by occlusive coronary thrombosis and treated with antiplatelet medications and primary percutaneous coronary intervention (PPCI). Pain is conventionally treated with opiates. Opiates may delay gastric transit and thus may reduce absorption of oral antiplatelet medication, which may impact on the ability of these drugs to reduce platelet reactivity. We sought to assess thrombotic state of STEMI patients receiving antiplatelet medications undergoing PPCI, according to morphine use.

Methods Patients with STEMI ( $\mathrm{n}=125)$ scheduled to undergo emergency PPCI, were assessed upon presentation (pre-PPCI) and again on day 2 of the admission after stabilisation on dual antiplatelet therapy. All patients were pre-treated with oral loading doses of $300 \mathrm{mg}$ aspirin and either ticagrelor $180 \mathrm{mg}(\mathrm{n}=23)$ or clopidogrel $600 \mathrm{mg}(\mathrm{n}=102)$ approximately 30-60 min before blood sampling. Thrombotic status was assessed by testing venous blood using the point-ofcare Global Thrombosis Test (GTT), which measures the time to form an occlusive thrombus under high shear (occlusion time, OT), and time to restore flow due to endogenous thrombolysis (lysis time, LT).

Results Patients treated with morphine ( $\mathrm{n}=101 ; 81 \%)$ exhibited enhanced platelet reactivity, as evidenced by shorter OT (mean $\pm \mathrm{SD} ; 358 \pm 143 \mathrm{~s}$ vs. $670 \pm 134 \mathrm{~s} ; \mathrm{p}<0.001$ ), and reduced thrombolytic potential, as evidenced by longer LT (median 1392s [IQR 1104-1864] vs. 1084s [875-1331]; p = 0.006) compared to those who had not received opiates. By day 2, no difference was apparent (OT: $485 \pm 142$ s vs. 552 $\pm 173 \mathrm{~s} ; \mathrm{p}=\mathrm{NS}$ and LT: 1322s [1187-1587] vs. 1184s [10511405]; $\mathrm{p}=\mathrm{NS}$ ) between the two groups.

Conclusions Patients receiving morphine analgesia before arrival to the catheterisation laboratory demonstrate enhanced platelet reactivity and impaired endogenous thrombolysis compared to those who did not, which may be attributable to reduced absorption of antiplatelet medications due to opiateinduced delayed gastric transit. This difference was no longer apparent by day 2 . Whether this impacts on recurrent early thrombosis risk, and whether non-opiate analgesia may proffer advantages in this setting, requires further assessment.

\section{VERIFY 2 (FINAL RESULTS): A COMPARISON OF FFR VS RESTING INDICES OF STENOSIS SEVERITY FOR DECISION MAKING IN THE CATH LAB. NCT02377310}

\footnotetext{
${ }^{1}$ Barry Hennigan*, ${ }^{1}$ Keith G Oldroyd, ${ }^{1}$ Colin Berry, ${ }^{1}$ Peter McCartney, ${ }^{2} J o h n$ McClure, ${ }^{1}$ Paul Rocchiccioli, ${ }^{1}$ Hany Eteiba, ${ }^{1}$ Richard Good, 'Martin M Lindsay, ${ }^{1}$ Stuart Hood, ${ }^{1}$ Margaret B McEntegart, 'Mark Petrie, 'Stuart Watkins. 'Golden Jubilee National Hosptial; ${ }^{2}$ University of Glasgow; *Presenting Author
}

\subsection{6/heartjnl-2016-309890.29}

Distal coronary to aortic pressure ratio $(\mathrm{Pd} / \mathrm{Pa})$ and instantaneous wave-free ratio (iFR) are resting indices of the functional significance of a coronary stenosis measured without inducing

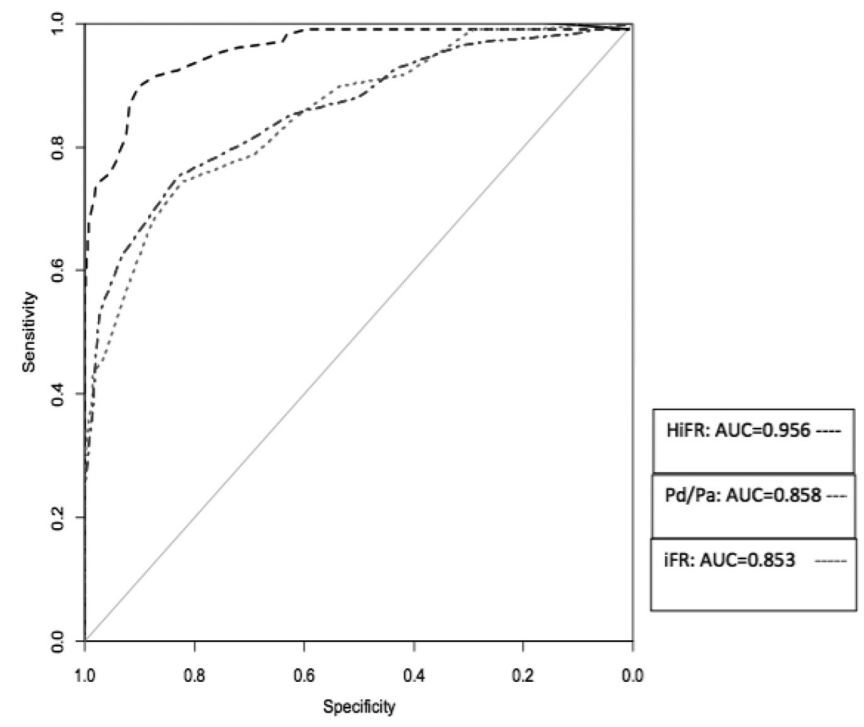

Abstract 29 Figure 1 Receiver operator characteristic curves demonstrating diagnostic accuracy of iFR, $\mathrm{Pd} / \mathrm{Pa}$ and HiFR in reference to FFR $<0.80$ Delong's test $p$-values for 2 correlated ROC curves: IFR vs iFR: 0.0001 , HiFR vs Pd/Pa: 0.0003

hyperaemia. It has been suggested that iFR has superior diagnostic accuracy to $\mathrm{Pd} / \mathrm{Pa}$ when both are compared to FFR. Hypotheses In comparison to an FFR for all strategy, revascularisation decisions based on either binary cut-off values for iFR or $\mathrm{Pd} / \mathrm{Pa}$ or hybrid strategies incorporating iFR or $\mathrm{Pd} / \mathrm{Pa}$ will result in similar levels of disagreement.

Methods A prospective study in consecutive patients undergoing FFR assessment for clinical indications using proprietary software to calculate iFR. We measured $\mathrm{Pd} / \mathrm{Pa}, \mathrm{iFR}, \mathrm{FFR}$ and hyperaemic iFR (HiFR). Diagnostic accuracy vs FFR was calculated firstly using binary cut-off values of $<0.90$ for iFR and $\leq 0.92$ for $\mathrm{Pd} / \mathrm{Pa}$ and again using the adenosine zones for iFR of $0.86-0.93$ and $\mathrm{Pd} / \mathrm{Pa}$ of $0.87-0.94$ in the hybrid strategy. The pre-determined sample size established prior to the start of the study was 254 vessels.

Results 197 patients with 257 moderate stenoses (mean DS 48\%) were studied. $127(49.4 \%)$ vessels were in patients with stable angina and $79(31 \%)$ vessels in patients with recent $(>72 \mathrm{~h})$ acute coronary syndromes. Using binary cut-off values diagnostic accuracy was similar for $\mathrm{iFR}$ and resting $\mathrm{Pd} / \mathrm{Pa}$ with misclassification rates of $20.6 \%$ vs $19.8 \%, p=0.86$. In the hybrid analysis, $54 \%$ of iFR cases and $53 \%$ of $\mathrm{Pd} / \mathrm{Pa}$ cases were outwith the adenosine zone. Rates of misclassification

Abstract 29 Table 1 Sensitivity analyses for iFr in proximal vs distal segments using $\leq 0.9$ cut-off compared with FFR. Proximal vs Distal - comparision of 2 proportions: $95 \% \mathrm{Cl}=(0.0248,0.225)$ Pearson Chi-sq test of assoc: $p=0.014$ Fisher's exact test: $p=$ 0.021

\begin{tabular}{|l|l|l|l|l|}
\hline Modality & Value & FFR $\leq 0.8$ & FFR $>0.8$ & Misclassification (\%) \\
\hline \multirow{2}{*}{$\begin{array}{l}\text { iFR proximal } \\
(1,11,5,6)\end{array}$} & $\leq 0.9$ & 33 & 15 & \multirow{2}{*}{27,7} \\
\cline { 2 - 5 } & $>0.9$ & 18 & 53 & \\
\hline \multirow{2}{*}{$\begin{array}{l}\text { iFR distal }(\neq \\
1,11,5,6)\end{array}$} & $\leq 0.9$ & 48 & 11 & \multirow{2}{*}{15.2} \\
\cline { 2 - 4 } & $>0.9$ & 10 & 63 & \\
\hline
\end{tabular}




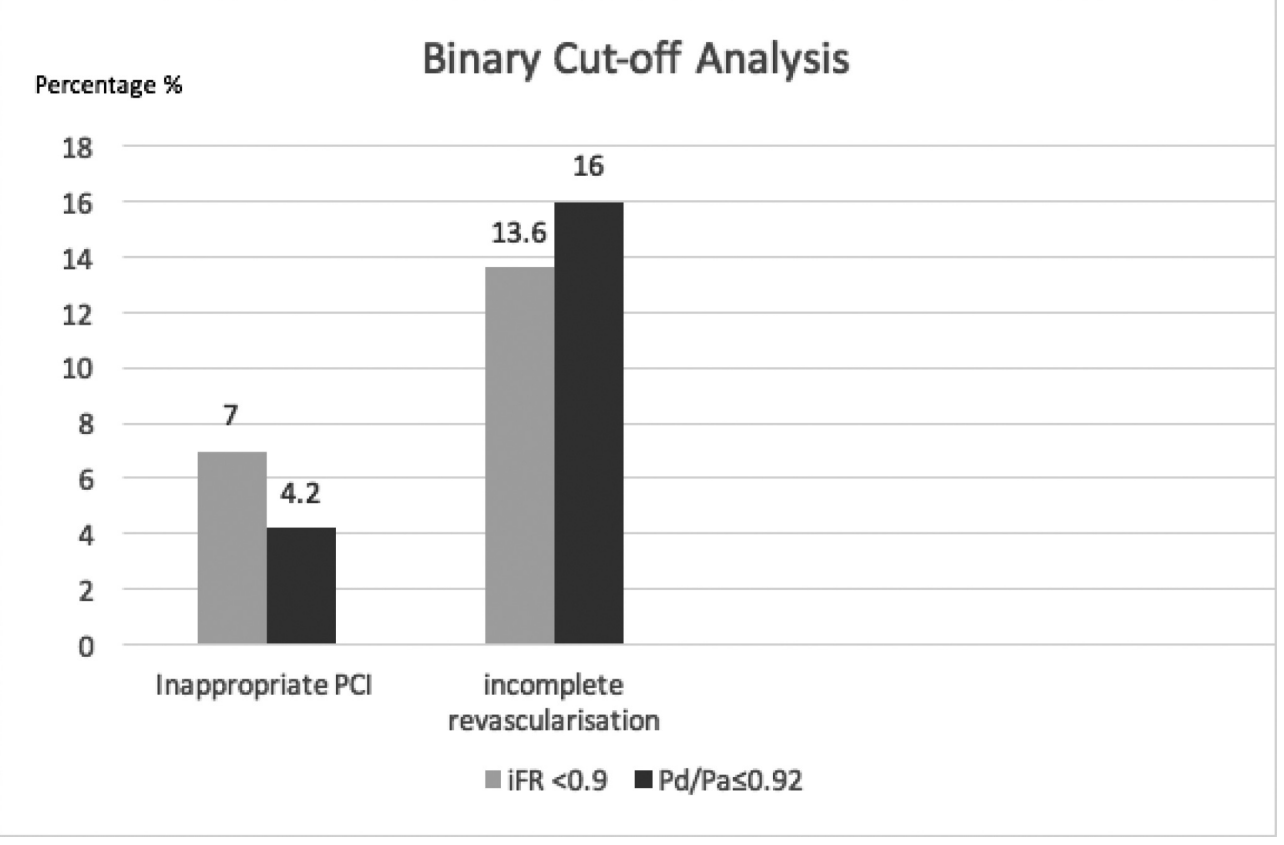

Abstract 29 Figure 2 Total number of inappropriate PCl's and incomplete revascularisations when binary cut-offs are utilised for Pd/Pa and iFR. Numbers shown are $\%$ of total, $n=257$

were $9.4 \%$ vs $11.9 \%, \mathrm{p}=0.55$. Sensitivity analyses showed no impact of a variety of angiographic measures of stenosis severity or myocardial area at risk. Comparing proximal stenoses (Syntax segments 1, 11, 5 and 6) to all other lesions and using the RESOLVE cutoff of $\leq 0.90$ for iFR the level of misclassification was $27.7 \%$ vs $15.2 \%, p=0.014$ (Table 1 ). Using the iFR cutoff of $<0.90$ the level of misclassification was $26.3 \%$ vs $16.2 \%, \mathrm{p}=0.05$.

Conclusion When compared to FFR, binary cut-offs for iFR and $\mathrm{Pd} / \mathrm{Pa}$ results in misclassification of 1 in 5 lesions. Using a hybrid strategy approximately half of the patient do not receive adenosine but 1 in 10 lesions is still misclassified. Neither resting index or strategy can be recommended for decision making in the cath lab. Operators wishing to use resting indices of stenosis severity should be particularly cautious when interpreting data from proximal stenoses in prognostically important vessels.

\section{HEAD-TO-HEAD COMPARISON OF TWO NOVEL INDICES OF MICROCIRCULATORY RESISTANCE AT PREDICTING MICROVASCULAR DYSFUNCTION. USE OF THE BEST INDEX TO EXPLORE THE EFFECT OF COLD AIR INHALATION DURING EXERCISE IN CORONARY ARTERY DISEASE PATIENTS}

${ }^{1}$ Rupert Williams* ${ }^{1}$ Kaleab Asrress, ${ }^{1}$ Matthew Lumley, ${ }^{2}$ Guus de Waord, ${ }^{1}$ Kalpa De Silva, 'Satpal Arri, ${ }^{1}$ Howard Ellis, ${ }^{1}$ Tiffany Patterson, ${ }^{1}$ Vasiliki Manou-Stathopoulou, 'Zeeshan Khawaja, ${ }^{1}$ Natalia Briceno, ${ }^{1}$ Brian Clapp, ${ }^{2}$ Sven Plein, ${ }^{3}$ Niels van Royen, ${ }^{1}$ Divaka Perera, ${ }^{1}$ Mike Marber, ${ }^{1}$ Simon Redwood. ${ }^{1}$ St Thomas' Hospital; ${ }^{3} \mathrm{VU}$ University Medical Center; ${ }^{2}$ Leeds University; ${ }^{*}$ Presenting Author

\subsection{6/heartjnl-2016-309890.30}

Introduction Highest rates of exertion related cardiac death occur during cold air inhalation (CAI): e.g. shovelling snow, but the pathophysiology is unclear. Novel intracoronary wires that simultaneously measure arterial pressure $\left(\mathrm{P}_{\mathrm{d}}\right)$ and blood flow (CBF) allow quantification of coronary micro-vascular resistance (MVR).

A. We compared the accuracy of Doppler-derived hyperemic micro-vascular resistance (hMR) and thermodilutionderived index of microcirculatory resistance (IMR) at predicting micro-vascular dysfunction, as there is no current invasive gold-standard measurement of MVR.

B. In coronary artery disease (CAD) patients we explored the effects of exercise with and without CAI on MVR (using the most accurate measure from A.) and CBF.

Methods A. 56 patients $(61+/-10$ years $)$ undergoing cardiac catheterization for stable $\mathrm{CAD}$ or acute myocardial infarction (AMI) were recruited. Simultaneous intracoronary pressure, Doppler flow velocity and thermodilution were carried out in 74 unobstructed vessels, at rest and during hyperemia. Three independent measures of micro-vascular function were assessed, using predefined dichotomous thresholds: 1) $\mathrm{CFR}_{\text {mean }}$, the average value of Doppler- and thermodilutionderived coronary flow reserve (CFR), and cardiovascular magnetic resonance derived 2) Myocardial Perfusion Reserve Index (MPRI) and 3) Micro-vascular Obstruction (MVO).

B. 35 CAD patients $(61+/-9$ years $)$ undertook $5 \mathrm{~min}$ of either:

1. CAI $\left(-15^{\circ} \mathrm{C}\right)$

2. Exercise (Incremental supine ergometry)

3. Exercise with CAI.

We measured baseline and peak MVR $\left(\mathrm{P}_{\mathrm{d}} / \mathrm{CBF}\right)$ and $\mathrm{CBF}$, and calculated the proportional contribution of waves that accelerate versus decelerate $\mathrm{CBF}$ as a coronary perfusion efficiency index.

Results A. hMR had better diagnostic accuracy than IMR to predict $\mathrm{CFR}_{\text {mean }}$ (area under curve, (AUC) 0.82 vs. $0.58, \mathrm{p}<$ 0.001, sensitivity/specificity $77 / 77 \%$ vs. $51 / 71 \%$ ) and MPRI (AUC 0.85 vs. $0.72, p=0.19$, sensitivity/specificity $82 / 80 \%$ vs. $64 / 75 \%$ ). In AMI patients, the AUCs of hMR and IMR at predicting MVO were 0.83 and 0.72 respectively $(p=0.22$, sensitivity/specificity $78 / 74 \%$ vs. $44 / 91 \%$ ). 